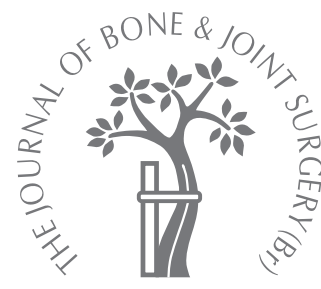

- CASE REPORT

\title{
Neglected trans-scaphoid trans-styloid volar dislocation of the lunate
}

\author{
LATE RESULT FOLLOWING OPEN REDUCTION AND \\ K-WIRE FIXATION
}

P. Givissis,

A. Christodoulou,

B. Chalidis,

J. Pournaras

From Aristotle

University of

Thessaloniki

"George

Papanikolaou”

Hospital,

Thessaloniki, Greece

\begin{abstract}
A rare case of radiocarpal dislocation is presented. The lunate and proximal pole of the scaphoid were displaced in a volar and proximal direction. The injury was missed initially and the patient was subsequently operated on six weeks later. Open reduction and internal fixation of the scaphoid was performed and this was followed by an uneventful postoperative period, with a satisfactory functional outcome at the eight-year follow-up, despite carpal instability non-dissociative-dorsal intercalated segmental instability configuration of the carpus.

We believe that although open reduction in neglected cases carries the potential risks of avascular necrosis and nonunion of the affected carpal bones, an attempt should be made to restore the anatomy of the carpus.
\end{abstract}

Trans-scaphoid volar dislocation of the lunate is a rare injury, which constitutes approximately $8 \%$ of all carpal dislocations. ${ }^{1}$ Only seven cases have reported volar dislocation of the lunate and the proximal pole of the scaphoid where the fragments were displaced proximal to the radiocarpal joint, ${ }^{2-6}$ the majority of which ${ }^{3-6}$ were treated quickly following injury.

The viability of the proximal pole of the scaphoid and the lunate is at risk following this type of injury. Reduced blood supply may result in nonunion of the scaphoid, avascular necrosis of the lunate or the proximal pole of the scaphoid, and instability of the wrist. ${ }^{2}$

Open reduction and internal fixation (ORIF) remains the treatment of choice. ${ }^{7}$ These injuries may not be recognised on initial examination. ${ }^{1}$ If early diagnosis is made, ORIF can provide satisfactory results. ${ }^{8,9}$ Late treatment of neglected injuries such as peri-lunate or lunate dislocation is difficult. ${ }^{10}$ The operative options available for the management of chronic dislocation include wrist fusion, proximal row carpectomy and excision of the lunate. ${ }^{9}$ There are few reports describing the management of unreduced fracture-dislocation of the carpus presenting six weeks after injury, ${ }^{11}$ and follow-up may be short. ${ }^{12}$ Most authors agree that open reduction and stabilisation are necessary ${ }^{10,11}$ and may be used up to 35 weeks following injury. ${ }^{2,13}$ If open reduction is not successful, reconstructive salvage procedures are required. ${ }^{10}$
We report the case of a patient who sustained a trans-scaphoid, trans-styloid volar dislocation of the lunate, in conjunction with the proximal pole of the scaphoid.

\section{Case report}

A 45-year-old right handed male manual labourer sustained an injury to his right wrist and a fracture of the first lumbar vertebra (L1) with paraparesis of the lower limbs, following a fall from a height of approximately $4.5 \mathrm{~m}$. The carpal injury was missed initially and he was referred six weeks later with discomfort while using his crutches and in everyday activities. The main priority had been the management of the lumbar fracture.

The wrist was deformed and tender, with limitation of movement and numbness in the distribution of the median nerve. Radiographs revealed a trans-scaphoid trans-styloid volar dislocation of the lunate, approximately $3 \mathrm{~cm}$ proximal to the radiocarpal joint (Fig. 1).

ORIF was undertaken through a palmar approach. The lunate and the proximal pole of the scaphoid remained united by an intact scapholunate ligament, but completely denuded of soft-tissue attachments, and were lying submuscularly in the forearm. Following the release of the carpal tunnel, the bones were reduced to their correct anatomical position. The scaphoid fracture was fixed with two 1.4 $\mathrm{mm}$ K-wires, which were placed perpendicular to the axis of the scaphoid, through the fracture line from a volar-proximal to a dorsal- 


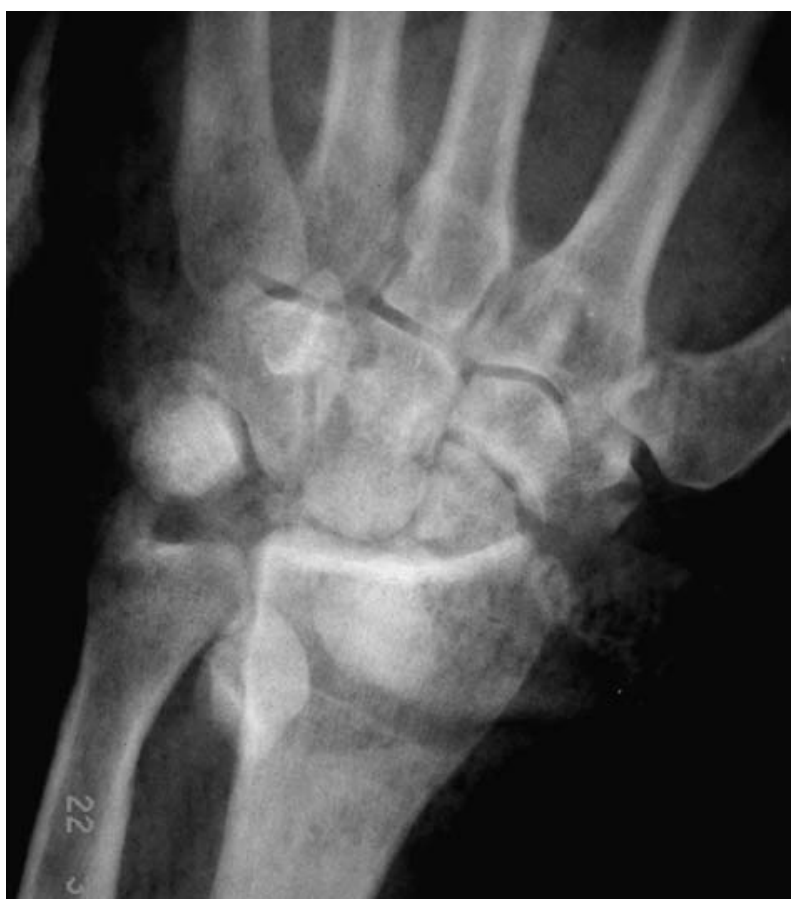

Fig. 1a

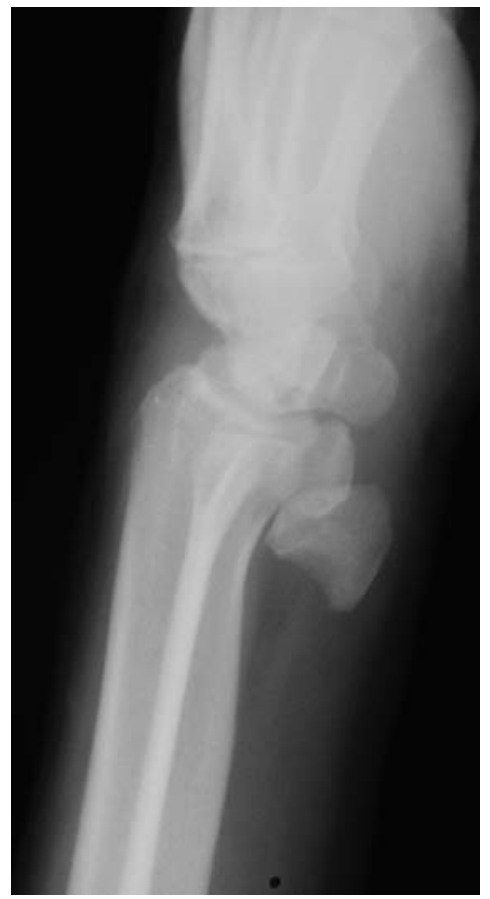

Fig. 1b

a) Anteroposterior and b) lateral radiographs showing trans-scaphoid trans-styloid volar dislocation of the lunate with proximal displacement.

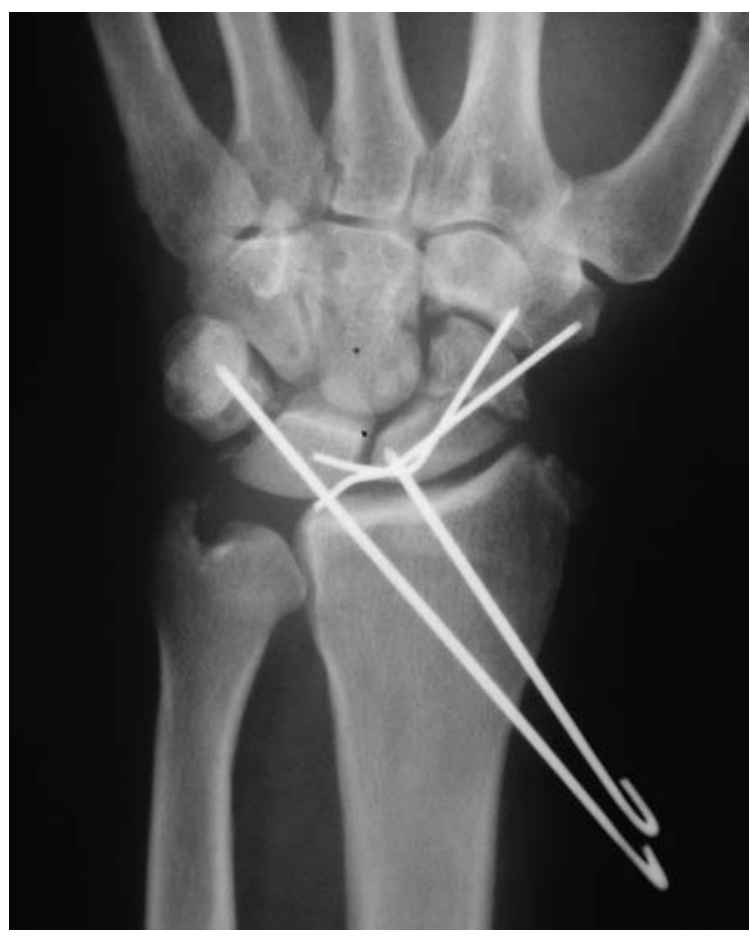

Fig. 2a

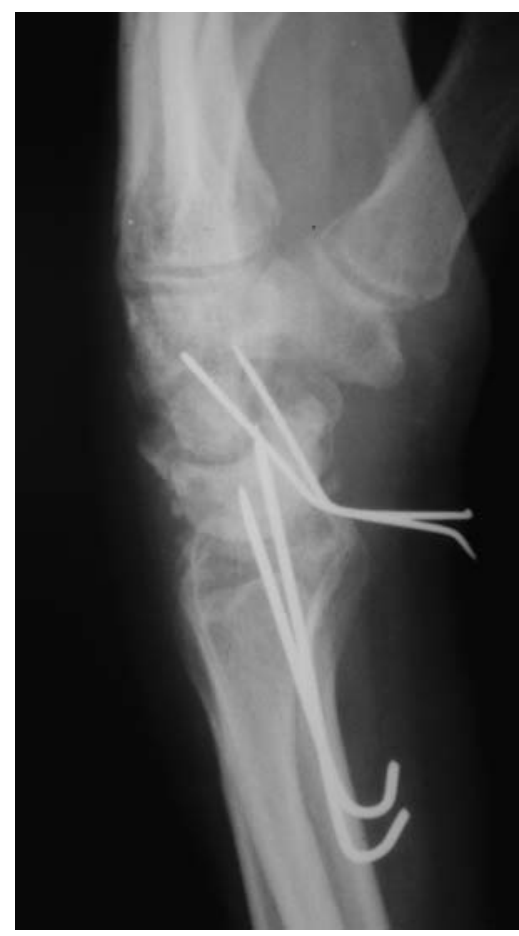

Fig. $2 b$

a) Anteroposterior and b) lateral intra-operative radiographs showing fixation of the scaphoid fracture and stabilisation of the carpus with K-wires. 


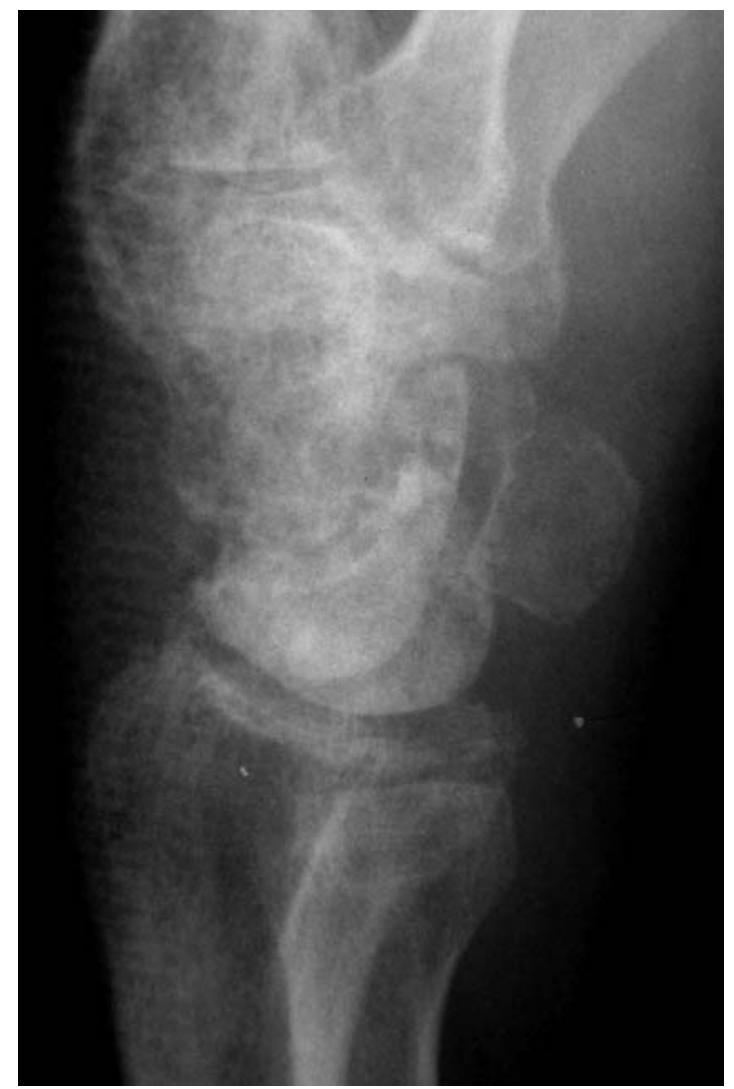

Fig. 3

Lateral radiograph six months post-operatively, showing the incipient carpal instability non-dissociative-dorsal intercalated segmental instability configuration of the carpus.

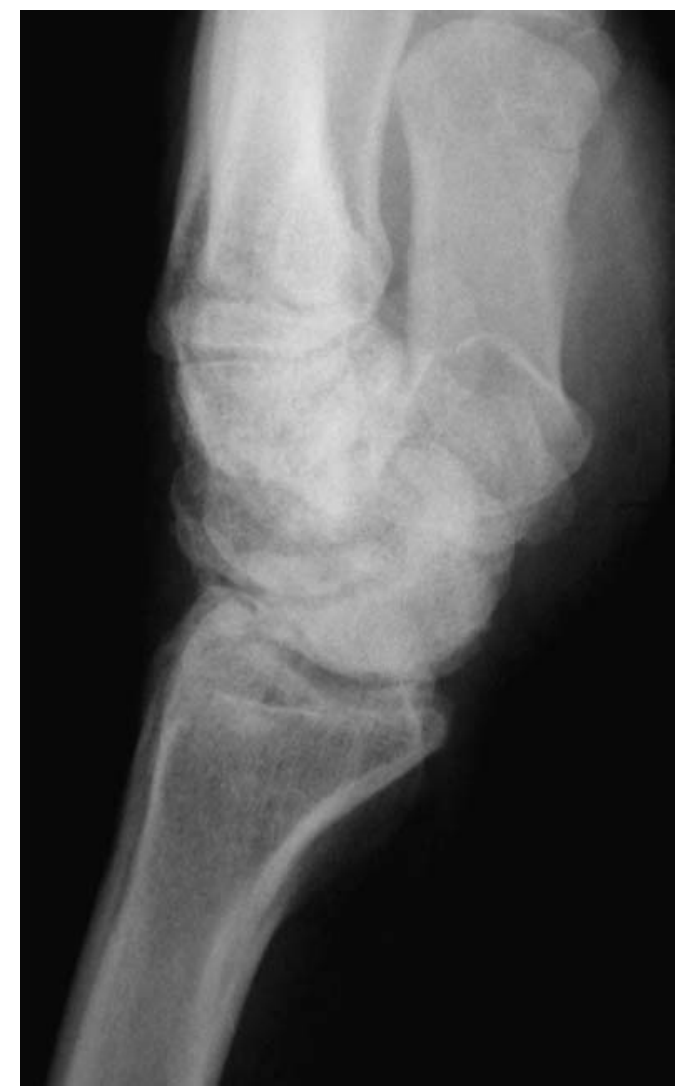

Fig. 4

Lateral radiograph eight years post-operatively, showing the progressive carpal instability non-dissociative-dorsal intercalated segmental instability configuration of the carpus. The capitolunate angle is abnormal, whilst the scapholunate angle has not changed. distal direction. The carpus was subsequently stabilised with two additional $2 \mathrm{~mm} \mathrm{~K}$-wires (Fig. 2). Because the dislocation had been left untreated for six weeks, the ligaments were attenuated and damaged so that primary repair could not be performed. The skin was closed over a drain and a below-elbow cast was applied.

The post-operative period was uneventful. The K-wires and the cast were removed after eight weeks and mobilisation of the wrist commenced. There was radiographic evidence of union of the scaphoid ten weeks post-operatively and he returned to his previous occupation within six months of injury. Radiographs showed incipient carpal instability non-dissociative-dorsal intercalated segmental instability (CIND-DISI) with an increased capitolunate angle, $>30^{\circ}$, while the scapholunate angle was normal (Fig. 3). This condition is associated with pathological extension of the lunate, as well as the scaphoid and triquetrum, with respect to the capitate and the radius. There is no abnormality of the scapholunate or lunotriquestral ligaments and the scapholunate and lunotriquetral angles are normal. ${ }^{14}$ At the eight-year follow-up visit, the degree of CIND-DISI had increased. The lateral view showed dorsal tilting of both the scaphoid and lunate, maintaining a scapholunate angle of approximately $70^{\circ}$, while the capitolunate angle was $>50^{\circ}$ (Fig. 4). In the anteroposterior view, there was loss of carpal height (carpal height ratio 0:44), a minimal ulnar shift of the carpus and early post-traumatic midcarpal and radiocarpal arthritis (Fig. 5). MRI scanning showed no evidence of avascular necrosis of the lunate or the proximal pole of the scaphoid (Fig. 6).

Radial and ulnar deviation of the wrist were $15^{\circ}$ and $20^{\circ}$ respectively, compared with $20^{\circ}$ and $35^{\circ}$ of the uninjured wrist. He lacked $10^{\circ}$ of volar flexion and $30^{\circ}$ of extension compared with the contralateral wrist. Pronation and supination of the forearm were equal bilaterally. Grip strength on the injured side, measured with the Jamar dynamometer (J. A. Preston, Jackson, Michigan), was 85\% when compared with the contralateral side. He was painfree and satisfied with the outcome and continued to work as a manual labourer.

\section{Discussion}

Volar dislocation of the lunate and the proximal pole of the scaphoid with displacement proximal to the radiocarpal 
joint, was first described by Green and O’Brien in $1978 .{ }^{2}$ It is a rare type of carpal dislocation. ${ }^{2-6}$

Closed reduction is technically difficult and most authors suggest that immediate ORIF followed by ligament repair (if possible) is the treatment of choice. ${ }^{12,15}$ However, Herzberg et $\mathrm{al}^{1}$ reported that $25 \%$ of peri-lunate dislocations are missed on examination for up to six weeks. The prognosis for these injuries with delayed presentation, is poor compared with those that are treated immediately. ${ }^{10,11,13}$ Open injuries, those inadequately reduced and those with nonunion have a poor outcome. , $^{1,12,16}$

The maximum possible time period after injury that a neglected peri-lunate or lunate dislocation may be reduced is unknown. ${ }^{10}$ Siegert et $\mathrm{al}^{13}$ described a series of patients who were treated by ORIF and had a satisfactory outcome, despite a time lapse between the injury and intervention and none required further surgery. Satisfactory results have also been reported following proximal row carpectomy in the treatment of chronic unreduced carpal dislocations ${ }^{17,18}$ and patients with marked displacement of the bones of the carpus and complete ligamentous disruption. ${ }^{19}$ Inoue and Shionoya ${ }^{17}$ and Rettig and Raskin ${ }^{18}$ noted that despite successful reduction, remnants of the ligament could not be repaired satisfactorily in order to prevent late carpal instability. Proximal row carpectomy should only be considered in patients with good quality articular cartilage, on both the head of the capitate and the lunate fossa of the radius. Partial or complete excision of the lunate is no longer recommended as a method of treatment. ${ }^{17}$

The risk of osteonecrosis developing in either the dislocated lunate or the proximal pole of the scaphoid has been recognised. ${ }^{2}$ There is potential for revascularisation of these bones, despite the marked displacement and the injury to the soft-tissue attachments. ${ }^{11}$ Panting et a ${ }^{20}$ noted osteonecrosis of either the lunate, or the proximal pole of the scaphoid in 12 of 27 patients with trans-scaphoid lunate or peri-lunate dislocation and reported revascularisation in nine. Ekerot ${ }^{3}$ hypothesised that revascularisation of the lunate takes place via the united fracture of the scaphoid and the intact scapholunate interosseous ligament. Furthermore, Gellman et $\mathrm{al}^{11}$ illustrated that the avascular changes of the lunate which developed following wrist dislocation may be transient.

In our case report, neither the scaphoid nor the lunate demonstrated any evidence of osteonecrosis, despite the delay in treatment, the extensive ligamentous damage and the poor blood supply to the injured area. An MRI scan showed no devascularisation of the bones of the carpus, or signs of scapholunate or lunotriquetral dissociation. Our findings are similar to those of some previously reported cases $^{3,4,6}$ where no osteonecrosis of the lunate or the scaphoid occurred. In other case reports nonunion of the scaphoid and osteonecrosis of the lunate and scaphoid have been described. ${ }^{2,4,5}$

The radiographs obtained eight years after surgery in our case study revealed a CIND-DISI configuration where the

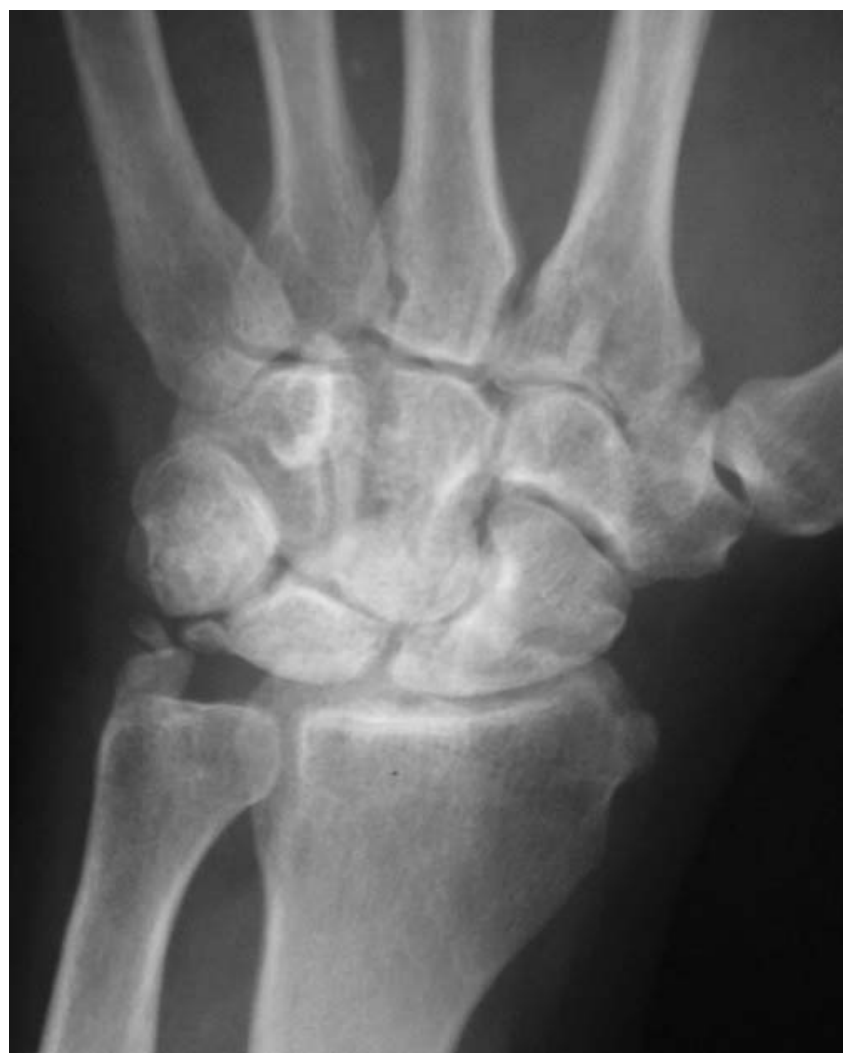

Fig. 5

Anteroposterior radiograph eight years post-operatively showing radiocarpal and midcarpal osteoarthritis, slight ulnar deviation of the carpus and loss of carpal height. The scaphoid is elongated and the lunate has a triangular shape, because of dorsal tilting.

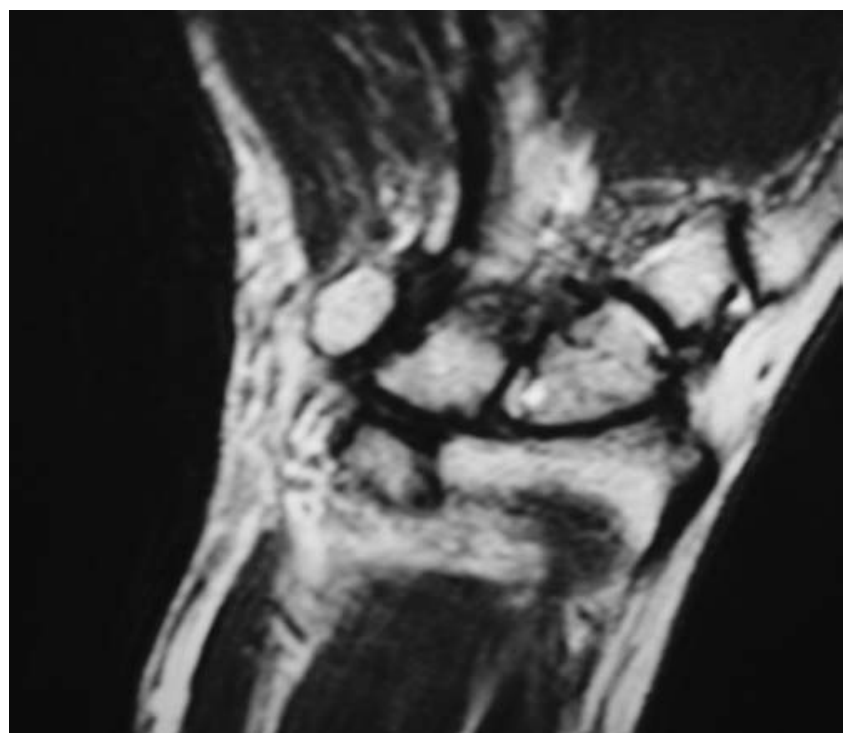

Fig. 6

An MRI scan (T1 image) at eight years follow-up, showing no evidence of avascular necrosis of the lunate or the proximal pole of the scaphoid. 
entire proximal carpal row had moved, without dissociation at the lunotriquetral or scapholunate joints. We believe that the delay in treatment led to the volar radiocarpal ligament deficiency which was possibly responsible for the partial wrist collapse.

Despite the CIND-DISI configuration of the wrist, the functional outcome of our patient was satisfactory, with a pain-free, good range of movement and a good grip strength. In addition, he was able to return to his occupation as a manual labourer. Similarly, Herzberg et $\mathrm{al}^{1}$ reported that unsatisfactory radiographs did not equate to a poor clinical outcome.

Although immediate management of peri-lunate dislocations is preferable for an optimal functional outcome, a satisfactory outcome can be achieved with open reduction and K-wire fixation, despite the potential risk of late carpal instability caused by nonunion of the scaphoid, necrosis of the lunate and poorly-healed carpal ligaments. Howard and Dell ${ }^{10}$ noted that if open reduction failed and only a partial reduction was obtained, it was generally better to see how the patient functioned, rather than performing an immediate reconstruction procedure. The recovery of our patient, who had a high risk of osteonecrosis because of the type of injury, suggests that late reduction and internal fixation is a viable option for the primary treatment of an unreduced carpal dislocation.

No benefits in any form have been received or will be received from a commercial party related directly or indirectly to the subject of this article.

\section{References}

1. Herzberg G, Comtet JJ, Linscheid RL, et al. Perilunate dislocations and fracturedislocations: a multicenter study. J Hand Surg [Am] 1993;18:768-79.

2. Green DP, O'Brien ET. Open reduction of carpal dislocations: indications and operative techniques. J Hand Surg [Am] 1978;3:250-65.
3. Ekerot L. Palmar dislocation of the trans-scaphoid-lunate unit. J Hand Surg [Br]1995; 20:557-60.

4. Stern PJ. Transcaphoid-lunate dislocation: a report of two cases. J Hand Surg [Am] 1984;9:370-3.

5. Stevanovic M, Schnall SB, Filler BC. Trans-scaphoid, transtriquetral, volar lunate fracture-dislocation of the wrist. J Bone Joint Surg [Am] 1996;78-A:1907-10.

6. Papadonikolakis A, Mavrodontidis A, Zalavras C, Hantes M, Soucacos P. Transscaphoid volar lunate dislocation: a case report. J Bone Joint Surg [Am] 2003; 85-A:1805-8

7. Adkison JW, Chapman MW. Treatment of acute lunate and perilunate dislocations. Clin Orthop 1982;164:199-207.

8. Inoue G, Imaeda T. Management of trans-scaphoid perilunate dislocations: Herbert screw fixation, ligamentous repair and early wrist mobilization. Arch Orthop Trauma Surg 1997;116:338-40.

9. Inoue G, Kuwahata Y. Management of acute perilunate dislocations without fracture of the scaphoid. J Hand Surg [Br] 1997;22:647-52.

10. Howard FM, Dell P. The unreduced carpal dislocation: a method of treatment. Clin Orthop 1986;202:112-16.

11. Gellman HG, Schwartz SD, Botte MJ, Feiwell L. Late treatment of a dorsal transscaphoid, transtriquetral perilunate wrist dislocation with avascular changes of the lunate. Clin Orthop 1988;237:196-203.

12. Moneim MS, Hofammann KE 3rd, Omer GE. Transscaphoid perilunate fracture-dislocation: result of open reduction and pin fixation. Clin Orthop 1984;190: 227-35.

13. Siegert JJ, Frassica FJ, Amadio PC. Treatment of chronic perilunate dislocations. J Hand Surg [Am] 1988;13:206-12.

14. Gilula LA, Mann FA, Dobyns JH, Yin Y. Wrist terminology as defined by the International Wrist Investigators' Workshop (IWIW). J Bone Joint Surg [Am] 2002;84-A (Suppl 1):1-66.

15. Cooney WP, Bussey R, Dobyns JH, Linscheid RL. Difficult wrist fractures: perilunate fracture-dislocations of the wrist. Clin Orthop 1987;214:136-47.

16. Viegas SF, Bean JW, Schram RA. Transscaphoid fracture/dislocations treated with open reduction and Herbert screw internal fixation. J Hand Surg [Am] 1987;12: 992-9.

17. Inoue G, Shionoya K. Late treatment of unreduced perilunate dislocations. J Hand Surg [Br] 1999;24:221-5.

18. Rettig ME, Raskin KB. Long-term assessment of proximal row carpectomy for chronic perilunate dislocations. J Hand Surg [Am] 1999;24:1231-6.

19. Draaijers LJ, Kreulen M, Maas M. Palmar dislocation of the lunate bone with complete disruption of all ligaments: a report of two cases. Acta Orthop Belg 2003; 69:452-4.

20. Panting AL, Lamb DW, Noble J, Haw CS. Dislocations of the lunate with and without fracture of the scaphoid. J Bone Joint Surg [Br] 1984;66-B:391-5. 